\title{
VERTEX-IRF TRANSFORMATIONS AND QUANTIZATION OF DYNAMICAL $r$-MATRICES
}

\author{
Pavel Etingof AND Dmitri Nikshych
}

\begin{abstract}
Motivated by the correspondence between the vertex and IRF models in statistical mechanics, we define and study a notion of vertex-IRF transformation for dynamical twists that generalizes a usual gauge transformation. We use vertexIRF transformations to quantize all completely degenerate dynamical $r$-matrices on finite-dimensional Lie algebras.
\end{abstract}

\section{Introduction}

This note has two goals. One is to describe the notion of a vertex-IRF transformation, which appeared in physics (as a map between the vertex and IRF models, see e.g., [Has]) and has so far remained a part of folklore, but which, we feel, is worthy of a systematic study. The other is to give examples of such transformations leading to quantization of dynamical $r$-matrices which have not been quantized previously.

Recall that the study of the dynamical quantum Yang-Baxter equation of Felder [F] leads one to a useful notion of a dynamical twist in a Hopf algebra (see [BBB, EV, ES2]). Namely, such a twist in a quasitriangular Hopf algebra gives rise to a solution of Felder's equation. There is a notion of a gauge transformation and gauge equivalence of dynamical twists; and twists which are gauge equivalent can be, in a sense, regarded as "the same" (e.g., the weak Hopf algebras associated to such twists as in [EN1] are isomorphic).

A vertex-IRF transformation is a generalization of a gauge transformation. More precisely, it is structurally similar to an ordinary gauge transformation, but unlike it, allows one to turn a known dynamical twist into an essentially new one. For example, one can apply vertex-IRF transformations to the identity twist $1 \otimes 1$ and obtain new dynamical twists. It turns out that in this way one can quantize a large class of dynamical $r$-matrices, which we call "completely degenerate". In some sense they are opposite to the non-degenerate dynamical $r$-matrices, which were quantized in $[\mathrm{X}]$.

The structure of the paper is as follows.

In Section 2 we define vertex-IRF transformations (with discrete and continuous dynamical variable), study their main properties, and then define vertex-IRF functors, which are a generalization of the functor between representation categories of the Felder and Belavin elliptic quantum groups discussed in [Has], [ES1].

Received March 13, 2001. 
In Section 3 we give examples of vertex-IRF transforms and resulting dynamical twists.

In Section 4 we introduce the notion of a completely degenerate dynamical $r$-matrix, and show that the methods of Section 3 allow one to quantize any such $r$-matrix. These quantizations appear to be new even in the simplest cases.

\section{Vertex-IRF transformations}

2.1. Definitions of vertex-IRF and IRF-vertex transformations. Let us give a rigorous definition of a vertex-IRF transformation. We will first give the definition for finite groups, and then generalize it to Lie groups.

We recall definitions of dynamical twists in Hopf algebras and their gauge equivalence from [EN2]. Let $H$ be a Hopf algebra with the comultiplication $\Delta$ and counit $\varepsilon$ over $\mathbb{C}$. Let $A \subset G(H)$ be a finite Abelian subgroup of the group $G(H)$ of group-like elements of $H$.

Notation 2.1. Let $P_{\mu}, \mu \in A^{*}$, be the set of primitive idempotents in $\mathbb{C}[A]$. Let $F: A^{*} \rightarrow H^{\otimes n}$ be a function. Set

$$
F\left(\lambda \pm h^{(i)}\right)=\sum_{\mu} F(\lambda \pm \mu) P_{\mu}^{i} \quad \text { and } \quad F\left(\lambda \pm \hat{h}^{(i)}\right)=\sum_{\mu} P_{\mu}^{i} F(\lambda \pm \mu),
$$

where $P_{\mu}^{i}=1 \otimes \cdots \otimes P_{\mu} \otimes \cdots \otimes 1$ with $P_{\mu}$ in the $i$ th component.

Definition 2.2. We say that an element $x$ in $H^{\otimes n}$ is of zero weight with respect to $A$ if $x$ commutes with $\Delta^{n}(a)$ for all $a \in A$, where $\Delta^{n}: H \rightarrow H^{\otimes n}$ is the iterated comultiplication.

Of course, $F\left(\lambda \pm h^{(i)}\right)=F\left(\lambda \pm \hat{h}^{(i)}\right)$ if $F$ is of zero weight with respect to the action of $A$ in the $i$ th component.

Definition 2.3. Let $J: A^{*} \rightarrow H \otimes H$ be a zero weight function with invertible values. We say that $J$ is a dynamical twist in $H$ if it satisfies the following functional equations

$$
\begin{gathered}
J^{12,3}(\lambda) J^{12}\left(\lambda-h^{(3)}\right)=J^{1,23}(\lambda) J^{23}(\lambda), \\
(\varepsilon \otimes \mathrm{id}) J(\lambda)=(\operatorname{id} \otimes \varepsilon) J(\lambda)=1 .
\end{gathered}
$$

Here $J^{12,3}(\lambda)=(\Delta \otimes$ id $) J(\lambda), J^{12}(\lambda)=J(\lambda) \otimes 1$ etc.

If $J$ is a dynamical twist in $H$ and $x: A^{*} \rightarrow H$ is a zero weight function with invertible values such that $\varepsilon(x(\lambda)) \equiv 1$, then

$$
J^{x}(\lambda)=\Delta(x(\lambda)) J(\lambda) x^{1}\left(\lambda-h^{(2)}\right)^{-1} x^{2}(\lambda)^{-1}
$$

is also a dynamical twist in $H$.

Definition 2.4. We say that $J^{x}$ is gauge equivalent to $J$ and that $x$ is a gauge transformation. 
Let $\bar{A}$ be a subgroup of $A, \bar{J}: \bar{A}^{*} \rightarrow H \otimes H$ be a dynamical twist, and $x: A^{*} \rightarrow H$ be a function with invertible values, of zero weight with respect to $\bar{A}$ (but not necessarily with respect to $A$ ), such that $\varepsilon(x(\lambda)) \equiv 1$. Define a function $J^{x}: A^{*} \rightarrow H \otimes H$ by the formula

$$
J^{x}(\lambda)=\Delta(x(\lambda)) \bar{J}(\bar{\lambda}) x^{2}(\lambda)^{-1} x^{1}\left(\lambda-h^{(2)}\right)^{-1},
$$

where $\lambda \mapsto \bar{\lambda}$ is the canonical projection $A^{*} \rightarrow \bar{A}^{*}$. Note that the order in which $x^{1}$ and $x^{2}$ are written is essential. Of course, $J^{x}(\lambda)$ is of zero weight with respect to $\bar{A}$, but a priori not with respect to $A$.

Proposition 2.5. If $J^{x}(\lambda)$ is of zero weight with respect to $A$, then it is a dynamical twist.

Proof. For all $\lambda \in A^{*}$ we compute, using the zero weight properties of $x(\lambda)$ and $J^{x}(\lambda)$ and the dynamical twist equation for $\bar{J}(\bar{\lambda})$ :

$$
\begin{aligned}
& J^{x 12,3}(\lambda) J^{x 12}\left(\lambda-h^{(3)}\right)= \\
& \quad=x^{123}(\lambda) \bar{J}^{12,3}(\bar{\lambda}) x^{3}(\lambda)^{-1} \bar{J}^{12}\left(\bar{\lambda}-\bar{h}^{(3)}\right) x^{2}\left(\lambda-h^{(3)}\right)^{-1} x^{1}\left(\lambda-h^{(2)}-h^{(3)}\right)^{-1} \\
& \quad=x^{123}(\lambda) \bar{J}^{12,3}(\bar{\lambda}) \bar{J}^{12}\left(\bar{\lambda}-\bar{h}^{(3)}\right) x^{3}(\lambda)^{-1} x^{2}\left(\lambda-h^{(3)}\right)^{-1} x^{1}\left(\lambda-h^{(2)}-h^{(3)}\right)^{-1}, \\
& J^{x 1,23}(\lambda) J^{x 23}(\lambda)= \\
& \quad=x^{123}(\lambda) \bar{J}^{1,23}(\bar{\lambda}) x^{23}(\lambda)^{-1} x^{1}\left(\lambda-h^{(2)}-h^{(3)}\right)^{-1} J^{x 23}(\lambda) \\
& \quad=x^{123}(\lambda) \bar{J}^{1,23}(\bar{\lambda}) \bar{J}^{23}(\bar{\lambda}) x^{3}(\lambda)^{-1} x^{2}\left(\lambda-h^{(3)}\right)^{-1} x^{1}\left(\lambda-h^{(2)}-h^{(3)}\right)^{-1} .
\end{aligned}
$$

The verification of equation (3) is straightforward.

Definition 2.6. If the assumption of Proposition 2.5 is satisfied, then the function $x$ is called a vertex-IRF transformation from $\bar{J}$ to $J^{x}$.

Proposition 2.5 has the following "converse" version.

Let $J: A^{*} \rightarrow H \otimes H$ be a dynamical twist, and $x: A^{*} \rightarrow H$ be a function with invertible values, of zero weight with respect to $\bar{A}$ (but not necessarily with respect to $A$ ), such that $\varepsilon(x)=1$. Let the function $J^{x}: A^{*} \rightarrow H \otimes H$ be given by the formula

$$
J^{x}(\lambda)=\Delta(x(\lambda)) J(\lambda) x^{1}\left(\lambda-h^{(2)}\right)^{-1} x^{2}(\lambda)^{-1}
$$

Proposition 2.7. If for some function $\bar{J}^{x}: \bar{A}^{*} \rightarrow H \otimes H$ we have $J^{x}(\lambda)=$ $\bar{J}^{x}(\bar{\lambda})$ for all $\lambda \in A^{*}$, then $\bar{J}^{x}$ is a dynamical twist. 
Proof. The computation below is similar to that of Proposition 2.5 and uses the zero weight properties of the functions involved :

$$
\begin{aligned}
& \bar{J}^{x 12,3}(\bar{\lambda}) \bar{J}^{x 12}\left(\bar{\lambda}-\bar{h}^{(3)}\right)= \\
& =x^{123}(\lambda) J^{x 12,3}(\lambda) x^{12}\left(\lambda-h^{(3)}\right)^{-1} \bar{J}^{x 12}\left(\bar{\lambda}-\bar{h}^{(3)}\right) x^{3}(\lambda)^{-1} \\
& =\quad x^{123}(\lambda) J^{x 12,3}(\lambda) J^{x 12}\left(\lambda-h^{(3)}\right) x^{1}\left(\lambda-h^{(2)}-h^{(3)}\right)^{-1} \\
& \quad \quad \times x^{2}\left(\lambda-h^{(3)}\right)^{-1} x^{3}(\lambda)^{-1}, \\
& \bar{J}^{x 1,23}(\lambda) \bar{J}^{x 23}(\lambda)= \\
& =x^{123}(\lambda) J^{x 1,23}(\lambda) x^{1}\left(\lambda-h^{(2)}-h^{(3)}\right)^{-1} J^{x 23}(\lambda) x^{2}\left(\lambda-h^{(3)}\right)^{-1} x^{3}(\lambda)^{-1} \\
& =x^{123}(\lambda) J^{x 1,23}(\lambda) J^{x^{23}}(\lambda) x^{1}\left(\lambda-h^{(2)}-h^{(3)}\right)^{-1} x^{2}\left(\lambda-h^{(3)}\right)^{-1} x^{3}(\lambda)^{-1} .
\end{aligned}
$$

The counit identity is straightforward.

Definition 2.8. If the assumption of Proposition 2.7 is satisfied, then $x$ is called an IRF-vertex transformation from $J$ to $\bar{J}^{x}$.

Remark 2.9. (1) Let $J, \bar{J}$ be dynamical twists defined on $A^{*}, \bar{A}^{*}$, respectively. Then $x: A^{*} \rightarrow H$ is a vertex-IRF transformation from $\bar{J}$ to $J$ if and only if $x^{-1}$ is an IRF-vertex transformation from $J$ to $\bar{J}$.

(2) If $A=\bar{A}$ then the assumptions of both propositions are vacuous, and a vertex-IRF or an IRF-vertex transformation is just a usual gauge transformation.

A useful special case of Proposition 2.5 is $\bar{A}=\{0\}$ when the twist to be transformed is $1 \otimes 1$. In this case, we have

Corollary 2.10. Let $x: A^{*} \rightarrow H$ be any invertible-valued function. If

$$
J(\lambda):=\Delta(x(\lambda)) x^{2}(\lambda)^{-1} x^{1}\left(\lambda-h^{(2)}\right)^{-1}
$$

is of zero weight then it is a dynamical twist.

Let us also describe how vertex-IRF and IRF-vertex transformations act on $R$-matrices. For this purpose, assume that $H$ is a quasitriangular Hopf algebra with the universal $R$-matrix $\mathcal{R}$ (see $[\mathrm{M}]$ for the definition). In this case, every dynamical twist $J(\lambda)$ defines the dynamical $R$-matrix $\mathcal{R}(\lambda)=J^{21}(\lambda)^{-1} \mathcal{R} J(\lambda)$, which satisfies the quantum dynamical Yang-Baxter equation.

Let now $J, \bar{J}$ be the twists on $A, \bar{A}$, and $x$ a vertex-IRF transformation such that $\bar{J}^{x}=J$. Let $\mathcal{R}, \overline{\mathcal{R}}$ be the dynamical $R$-matrices associated to $J, \bar{J}$.

Corollary 2.11. The $R$-matrices above are related by

$$
\mathcal{R}(\lambda)=x^{2}\left(\lambda-h^{(1)}\right) x^{1}(\lambda) \overline{\mathcal{R}}(\bar{\lambda}) x^{-2}(\lambda) x^{-1}\left(\lambda-h^{(2)}\right) .
$$

Let us now extend the above theory to the case of Hopf algebras over $\mathbb{C}[[\hbar]]$. Let $H$ be a deformation Hopf algebra over $\mathbb{C}[[\hbar]]$ (for example, a quantized universal enveloping algebra), and let $H_{0}=H / \hbar H$. Let $\mathfrak{a}$ be a finite-dimensional (over $\mathbb{C}$ ) commutative Lie subalgebra of $\operatorname{Prim}(H)$, the Lie algebra of primitive elements of $H$, such that the induced map $\mathfrak{a} \rightarrow \operatorname{Prim}\left(H_{0}\right)$ is injective. In this situation, we make the following definition. 
Definition 2.12. A zero weight $H \otimes H$-valued meromorphic function $J(\lambda)$ on $\mathfrak{a}^{*}$ is called a formal dynamical twist for $H$ if it equals $1 \otimes 1$ modulo $\hbar$ and satisfies the following functional equations :

$$
\begin{gathered}
J^{12,3}(\lambda) J^{12}\left(\lambda-\hbar h^{(3)}\right)=J^{1,23}(\lambda) J^{23}(\lambda), \\
(\varepsilon \otimes \mathrm{id}) J(\lambda)=(\mathrm{id} \otimes \varepsilon) J(\lambda)=1 .
\end{gathered}
$$

Here the expression $J^{12}\left(\lambda-\hbar h^{(3)}\right)$ is understood in the sense of the Taylor expansion with respect to $\hbar$.

Note that this definition differs from Definition 2.3 only by replacing $h^{(3)}$ with $\hbar h^{(3)}$. Because of this, in the sequel we will drop the word "formal", and refer to $J$ as a "dynamical twist".

To define vertex-IRF and IRF-vertex transformations in the formal situation, we will define the group $\tilde{H}^{\times}$, which is an extension by the reduced multiplicative group $1+\hbar H$ of $H$ of the simply connected Lie group $G$ corresponding to the Lie algebra $\mathfrak{g}$ of primitive elements of $H_{0}$. To make this definition, we will assume that

(1) the Lie algebra $\mathfrak{g}$ is finite-dimensional, and

(2) the $\mathfrak{g}$ module $H_{0}$ (where the action is by the commutator) is a sum of finite-dimensional submodules.

These conditions are satisfied, for example, if $H_{0}$ is the enveloping algebra of a finite-dimensional Lie algebra.

Consider the Lie algebra $\mathfrak{l}$ of all elements $y \in H$ such that $y \in \mathfrak{g} \bmod \hbar$ (under commutator). We have an exact sequence of Lie algebras

$$
0 \rightarrow \hbar H \rightarrow \mathfrak{l} \rightarrow \mathfrak{g} \rightarrow 0
$$

It is easy to show that under assumptions (1) and (2), the Lie algebra $\mathfrak{l} / \hbar^{n} \mathfrak{l}$ is a sum of finite-dimensional Lie algebras. This implies that the above exact sequence of Lie algebras canonically defines an exact sequence of groups

$$
0 \rightarrow 1+\hbar H[[\hbar]] \rightarrow L \rightarrow G \rightarrow 0 .
$$

This defines the desired group $L$, which we will denote by $\tilde{H}^{\times}$. We have an exponential map exp $: \mathfrak{l} \rightarrow L$, which allows us to think of $\mathfrak{l}$ as a Lie algebra of $L$. In other words, one may think of (small) elements of $\tilde{H}^{\times}$as expressions of the form $e^{F}$, where $F \in H$ and $F \bmod \hbar \in \mathfrak{g}$ (in these terms, the multiplication in $\tilde{H}^{\times}$uses the Campbell-Hausdorff formula).

Now let $\overline{\mathfrak{a}}$ be a Lie subalgebra of $\mathfrak{a}$.

We will make the following modifications in the above theory of vertex-IRF transformations:

1. $A, \bar{A}$ are replaced by $\mathfrak{a}, \overline{\mathfrak{a}}$.

2. The functions $x(\lambda)$ are required to be meromorphic and take values in $\tilde{H}^{\times}$.

3. In all formulas $h^{(i)}$ is replaced with $\hbar h^{(i)}$.

Then we have 
Proposition 2.13. Propositions 2.5, 2.7 and Corollaries 2.10, 2.11 are valid in the formal case with the above modifications.

Remark 2.14. Let us motivate the terminology "vertex-IRF" and "IRF-vertex" transformation. The point is that formula (7) is (up to small modifications) exactly the same as the formula representing the relationship between the $R$ matrices of the 8-vertex and the interaction-round-a-face models of statistical mechanics, discovered by Baxter (for $n=2$ ) and developed by Hasegawa [Has] (see also [ES1, Lemma 2]). In fact, it is known by now (see [JOKS]) that these $R$-matrices (namely, the Baxter-Belavin and the Felder $R$-matrices in the vector representation) can be obtained by specializing universal $R$-matrices obtained from the usual trigonometric $R$-matrix of the quantum affine algebra $U_{q}(\widehat{s l(n)})$ by twisting using a non-dynamical and a dynamical twist, respectively. It is expected that these two twists in fact are related by a vertex-IRF transformation in $U_{q}(\widehat{s l(n)})$; however, the universal expression for this transformation is not known: it is only known in the vector representation, and in a tensor product of shifted vector representations (see [ES1]). The universal expression is known, however, for $n=2$ in a trigonometric degeneration (see [BBB], the element $M(\lambda)$ ). The case $n>2$ was intensively studied by E. Buffenoir and Ph. Roche (unpublished), who turned the authors' attention to the notion of the vertex-IRF transformation. We also note that the vertex-IRF transformation for $g l(n)$ (in the vector representation), was, in effect, used by Cremmer and Gervais in their proof that the Cremmer-Gervais $R$-matrix satisfies the quantum Yang-Baxter equation. This was explained by Hodges in [Ho].

2.2. An IRF-vertex functor. In this subsection we would like to explain how a vertex-IRF transformation between two dynamical twists gives rise to a certain functor between the corresponding categories of representations. For the original vertex-IRF transformation coming from physics, this functor was essentially introduced by Hasegawa (see [Has]) and was studied in [ES1].

For simplicity we will deal with the situation when the dynamical twist is defined on the dual of a finite abelian group $A$ lying inside a finite-dimensional Hopf algebra $H$; the cases of a finite-dimensional Lie algebra $\mathfrak{a}$ instead of $A$ and of infinite dimensional $H$ are completely parallel.

Let $J: A^{*} \rightarrow H \otimes H$ be a dynamical twist. Then one can define the category $\operatorname{Rep}(J)$ of representations of the dynamical quantum group associated to $J$. This can be done, for instance, using weak Hopf algebras introduced in [BNSz]. Namely, one can define a weak Hopf algebra $H^{J}(H$ twisted by $J)$ as in [EN1], and $\operatorname{Rep}(J)$ is nothing but the category of its comodules.

Let us also give another, more explicit description of $\operatorname{Rep}(J)$, which can be found in [EV, ES1]. Apart from being more explicit, this description has the advantage that, unlike the weak Hopf algebra description, it can be easily adapted to the case when $A$ is replaced with an abelian Lie algebra $\mathfrak{a}$.

An object of $\operatorname{Rep}(J)$ is an $A$-module $V$, together with an assignment $X \rightarrow$ $L_{X}(\lambda)$, which assigns to any $H$-module $X$ the L-operator of this module, $L_{X}$ : 
$A^{*} \rightarrow \operatorname{End}_{A}(X \otimes V)$. This assignment is required to be compatible with morphisms in $\operatorname{Rep}(H)$ and to be compatible with tensor products in the following way:

$$
J^{12}\left(\lambda-h^{(3)}\right) L_{Y}^{23}(\lambda) L_{X}^{13}\left(\lambda-h^{(2)}\right) J^{12}(\lambda)^{-1}=L_{X \otimes Y}
$$

in $X \otimes Y \otimes V$.

A morphism between representations $(V, L),\left(V^{\prime}, L^{\prime}\right)$ is a function $f: A^{*} \rightarrow$ $\operatorname{Hom}_{A}\left(V, V^{\prime}\right)$ such that

$$
(1 \otimes f(\lambda)) L_{X}(\lambda)=L_{X}^{\prime}(\lambda)\left(1 \otimes f\left(\lambda-h^{(1)}\right)\right) .
$$

Now we proceed to construct the desired functor. Let $A, J$ be as above and $\bar{J} \in H \otimes H$ be an ordinary twist. Assume that $x: A^{*} \rightarrow H$ is a vertex-IRF transformation from $\bar{J}$ to $J$.

Let $(V, L) \in \operatorname{Rep}(J)$. For any $X \in \operatorname{Rep}(H)$ define the linear operator $\bar{L}_{X}$ on the vector space $X \otimes V \otimes F\left(A^{*}\right)$, where $F\left(A^{*}\right)$ denotes the function algebra on $A^{*}$ :

$$
\bar{L}_{X}:=x^{1}\left(\lambda-h^{(2)}\right)^{-1} \circ L_{X}(\lambda) \circ T_{1} \circ x^{1}\left(\lambda+\hat{h}^{(1)}\right) .
$$

Here we use Notation 2.1 and set $T_{1} f(\lambda) x \otimes v:=f\left(\lambda-h^{(1)}\right) x \otimes v$.

The main result of this subsection is

Theorem 2.15. (i) The operators $\bar{L}_{X}$ turn the vector space $V \otimes F\left(A^{*}\right)$ into an object of $\operatorname{Rep}(\bar{J})$ (i.e., a comodule over the Hopf algebra $\left.H^{\bar{J}}\right)$.

(ii) The assignment $\mathcal{F}:(V, L) \mapsto\left(V \otimes F\left(A^{*}\right), \bar{L}\right)$ is a functor.

Remark 2.16. We note that here we have considered only the situation $\bar{A}=0$. If $\bar{A} \neq 0$ and $A=\bar{A} \oplus \bar{A}^{\prime}$ then one can generalize the theorem and construct a functor $\mathcal{F}$ which, at the level of vector spaces, reduces to tensoring with $F\left(\left(\bar{A}^{\prime}\right)^{*}\right)$.

Proof. The statements are proved by direct verification. We will only prove the more difficult statement (i). One has

$$
\begin{aligned}
\bar{L}_{X \otimes Y}= & x^{12}\left(\lambda-h^{(3)}\right)^{-1} L_{X \otimes Y}(\lambda) T_{1} T_{2} x^{12}\left(\lambda+\hat{h}^{(1)}+\hat{h}^{(2)}\right) \\
= & x^{12}\left(\lambda-h^{(3)}\right)^{-1} J^{12}\left(\lambda-h^{(3)}\right) L_{Y}^{23}(\lambda) L_{X}^{13}\left(\lambda-h^{(2)}\right) J^{12}(\lambda)^{-1} \\
& \quad \times T_{1} T_{2} x^{12}\left(\lambda+\hat{h}^{(1)}+\hat{h}^{(2)}\right) \\
= & \bar{J}^{12} x^{2}\left(\lambda-h^{(3)}\right)^{-1} x^{1}\left(\lambda-h^{(2)}-h^{(3)}\right)^{-1} L_{Y}^{23}(\lambda) L_{X}^{13}\left(\lambda-h^{(2)}\right) \\
& \quad \times T_{1} T_{2} J^{12}\left(\lambda+h^{(2)}+h^{(3)}\right)^{-1} x^{12}\left(\lambda+\hat{h}^{(1)}+\hat{h}^{(2)}\right) \\
= & \bar{J}^{12} x^{2}\left(\lambda-h^{(3)}\right)^{-1} L_{Y}^{23}(\lambda) x^{1}\left(\lambda-h^{(2)}-h^{(3)}\right)^{-1} L_{X}^{13}\left(\lambda-h^{(2)}\right) \\
& \quad \times T_{1} T_{2} x^{2}\left(\lambda+\hat{h}^{(1)}+\hat{h}^{(2)}\right) x^{1}\left(\lambda+\hat{h}^{(1)}\right)\left(\bar{J}^{12}\right)^{-1} \\
= & \bar{J}^{12} x^{2}\left(\lambda-h^{(3)}\right)^{-1} L_{Y}^{23}(\lambda) T_{2} x^{2}\left(\lambda+\hat{h}^{(2)}\right) x^{1}\left(\lambda-h^{(3)}\right)^{-1} L_{X}^{13}(\lambda) \\
& \quad \times T_{1} x^{1}\left(\lambda+\hat{h}^{(1)}\right)\left(\bar{J}^{12}\right)^{-1} \\
= & \bar{J}^{12} \bar{L}_{Y}^{23} \bar{L}_{X}^{13}\left(\bar{J}^{12}\right)^{-1}
\end{aligned}
$$

as desired. 
The functor $\mathcal{F}$ is quite nontrivial. For example, let $V$ be the trivial representation $(V=\mathbb{C}, L=1)$. Then $\mathcal{F}(V)=F\left(A^{*}\right)$, with the action of $\left(H^{J}\right)^{*}$ given by:

$$
L_{X}=x^{1}(\lambda)^{-1} T_{1} x^{1}\left(\lambda+\hat{h}^{(1)}\right) .
$$

This is typically a very nontrivial representation of $\left(H^{J}\right)^{*}$ by scalar difference operators on $A^{*}$. For example, for the vertex-IRF transformation in statistical mechanics this very interesting representation was considered by Hasegawa; it is connected to the theory of integrable systems, see [KZ].

\section{On classification and examples of vertex-IRF transformations}

Now let us consider the special case when $\bar{A}=\{0\}$. We will be interested in finding vertex-IRF transformations of the twist $1 \otimes 1$. That is, we want to find functions $x(\lambda)$ such that

$$
(1 \otimes 1)^{x}=\Delta(x(\lambda)) x^{2}(\lambda)^{-1} x^{1}\left(\lambda-h^{(2)}\right)^{-1}
$$

is a dynamical twist (i.e., is of zero weight).

We note, first of all, that the set $\mathcal{S}$ of such functions carries an action of the group of gauge transformations $\mathcal{G}$ of dynamical twists $A^{*} \rightarrow H \otimes H$, since one can always compose a vertex-IRF transformation with a gauge transformation and obtain a new vertex-IRF transformation. What we are really interested in is the description of the set of orbits, $\mathcal{S} / \mathcal{G}$.

Below we formulate a theorem which describes all elements of $\mathcal{S}$ which normalize $\mathbb{C}[A]$. Let $N_{A}$ be the normalizer of $\mathbb{C}[A]$ in the multiplicative group $H^{\times}$. We have a natural homomorphism from $N_{A}$ to the group of permutations of $A^{*}$, $\pi: N_{A} \rightarrow \operatorname{Perm}\left(A^{*}\right)$, defined by the action of $N_{A}$ on the primitive idempotents of $\mathbb{C}[A]$.

Theorem 3.1. A function $x: A^{*} \rightarrow N_{A}$ is a vertex-IRF transformation of $1 \otimes 1$ if and only if

$$
\pi(x(\lambda))^{-1}(\mu)=f(\lambda)-f(\lambda-\mu),
$$

for a suitable bijective function $f: A^{*} \rightarrow A^{*}$. Furthermore, two such vertex-IRF transformations $x_{i}, i=1,2$, are gauge equivalent if and only if they define the same permutations $\pi\left(x_{i}(\lambda)\right)$.

Proof. Let $\pi(x(\lambda))^{-1}(\mu)=F_{\lambda}(\mu)$. It is easy to see that the condition for $x$ to be a vertex-IRF transformation is

$$
F_{\lambda}(a+b)=F_{\lambda}(b)+F_{\lambda-b}(a),
$$

for all $a, b \in A^{*}$. Setting $b=0$ one gets $F_{\lambda}(0)=0$. Also, setting $b=\lambda$, we find

$$
F_{\lambda}(a+\lambda)=f(\lambda)-g(a)
$$

where $f(\lambda)=F_{\lambda}(\lambda)$ and $g(a)=F_{0}(a)$. Putting in the last equation $a=-\lambda$, we find $g(a)=f(-a)$, i.e.

$$
F_{\lambda}(a+\lambda)=f(\lambda)-f(-a)
$$


as desired. The converse statement is straightforward.

In the situation of this theorem, we will say that $x$ realizes the function $f$.

Remark 3.2. Theorem 3.1 shows that the set of vertex-IRF transformations normalizing $\mathbb{C}[A]$, modulo gauge equivalences, is finite. They, however, do not exhaust all the variety of vertex-IRF transformations; in particular, the original vertex-IRF transformations coming from physics are not of this type.

For general $H$, it is not easy to find all functions $f: A^{*} \rightarrow A^{*}$ realized by vertex-IRF transformations. Below we use the classification results of [EN2] to derive an answer in the case when $H=\mathbb{C}[G]$, where $G$ is a finite group.

Recall [EN2, Theorem 6.6] that the gauge equivalence classes of dynamical twists $J(\lambda): A^{*} \rightarrow \mathbb{C}[G] \otimes \mathbb{C}[G]$ are in bijective correspondence with isomorphism classes of dynamical data for $(G, A)$, i.e., collections $\left(K,\left\{V_{\lambda}\right\}_{\lambda \in A^{*}}\right)$, where $K$ is a subgroup of $G$ and $V_{\lambda}$ are irreducible projective representations of $K$ such that $V_{\lambda} \otimes V_{\mu}^{*}$ is linear and

$$
\operatorname{Ind}_{K}^{G}\left(V_{\lambda} \otimes V_{\mu}^{*}\right) \cong \operatorname{Ind}_{A}^{G}(\lambda-\mu)
$$

for all $\lambda, \mu \in A^{*}$ (see [EN2, Section 4] for a detailed discussion of this notion).

Proposition 3.3. The functions $f$ which are realized by vertex-IRF transformations are those for which

$$
\operatorname{Ind}_{A}^{G}(\lambda-\mu) \cong \operatorname{Ind}_{A}^{G}(f(\lambda)-f(\mu))
$$

for all $\lambda, \mu \in A^{*}$. In particular, a dynamical twist is obtained from $1 \otimes 1$ by a vertex-IRF transformation if and only if in the dynamical data corresponding to it, one has $K=A$.

Proof. If $f$ is realized by a vertex-IRF transformation $x$, then for all $\lambda, \mu \in A^{*}$ the characters $\lambda-\mu$ and $f(\lambda)-f(\mu)$ are conjugate via $\operatorname{Ad}_{\pi(x(\lambda))^{-1}}$, and therefore induce equivalent representations of $G$.

Conversely, if (13) holds for a bijective function $f: A^{*} \rightarrow A^{*}$ then the collection $\left(A,\left\{\mathbb{C}_{f(\lambda)}\right\}_{\lambda \in A^{*}}\right)$ is a dynamical datum for $(G, A)$. For every element $y \in \mathbb{C}[G]$ of weight $\mu$ the homomorphism $\Psi(\lambda, y): \mathbb{C}_{f(\lambda)} \rightarrow \mathbb{C}_{f(\lambda-\mu)} \otimes \mathbb{C}[G]$ arising in the exchange construction [EN2, Section 6], is given by $1 \mapsto 1 \otimes x(\lambda) y$, where the element $x(\lambda)$ belongs to the normalizer of $\mathbb{C}[A]$ in $\mathbb{C}[G]^{\times}$. In every $G$-module $x(\lambda)$ maps elements of weight $\mu$ to elements of weight $f(\lambda)-f(\lambda-\mu)$, for all $\lambda, \mu \in A^{*}$. Clearly, $x(\lambda)$ realizes $f$ and the twist defined by the above action is equal to $(1 \otimes 1)^{x}$.

As an example, consider a class of vertex-IRF transformations in $\mathbb{C}[G]$ defined by elements $x$ which may be called "quasi-grouplike elements" (this example was considered in [EN2]).

Proposition 3.4. Let $g: A^{*} \times A^{*} \rightarrow G$ be a function, such that

$$
(\lambda-\mu) \circ A d_{g(\lambda, \mu)}=f(\lambda)-f(\mu) .
$$

Then 
(i) $x(\lambda):=g\left(\lambda, \lambda-h^{(1)}\right)$ is a vertex-IRF transformation of $1 \otimes 1$ realizing $f$.

(ii) The transformed dynamical twist has the form

$$
\begin{aligned}
& J(\lambda)=: g\left(\lambda, \lambda-h^{(1)}-h^{(2)}\right) g^{-1}\left(\lambda-h^{(2)}, \lambda-h^{(1)}-h^{(2)}\right) \otimes \\
& \otimes g\left(\lambda, \lambda-h^{(1)}-h^{(2)}\right) g^{-1}\left(\lambda, \lambda-h^{(2)}\right):
\end{aligned}
$$

where the colons on both sides mean "normal ordering" : elements $h^{(1)}$ and $h^{(2)}$ are put to the extreme right, i.e., they are replaced by $\mu, \nu$ respectively when the twist acts on a vector $v \otimes w$ such that $v, w$ have weights $\mu, \nu$.

Proof. The proof is contained in [EN2, Example 6.10].

The theory developed in this Section can be extended to the case when the finite group $A$ is replaced by an abelian Lie algebra $\mathfrak{a}$, along the lines described in Section 2. We will not do it completely, but will just show how the generalization of the Proposition 3.4 allows one to quantize some classical dynamical $r$-matrices.

Let $\mathfrak{g}$ be a finite-dimensional Lie algebra over $\mathbb{C}$ with an Abelian Lie subalgebra $\mathfrak{a}$. Let $G$ be the corresponding Lie group. Let $f: \mathfrak{a}^{*} \rightarrow \mathfrak{a}^{*}$ be a meromorphic mapping.

We have the following analogue of Proposition 3.4. Let $g: \mathfrak{a}^{*} \times \mathfrak{a}^{*} \rightarrow G$ be a meromorphic function well defined at generic points of the diagonal (in fact, it is only needed that $g$ is defined in the formal neighborhood of the diagonal).

Proposition 3.5. Assume that for all $\lambda, \mu \in \mathfrak{a}^{*}$ we have

$$
(\lambda-\mu) \circ A d_{g(\lambda, \mu)}=f(\lambda)-f(\mu) .
$$

Then

(i) $x(\lambda):=g\left(\lambda, \lambda-\hbar h^{(1)}\right)$ is a vertex-IRF transformation of $1 \otimes 1$ realizing $f$, i.e., $\pi(x(\lambda))^{-1}(\mu)=\hbar^{-1}(f(\lambda)-f(\lambda-\hbar \mu))$.

(ii) The transformed dynamical twist has the form

$$
\begin{array}{r}
J(\lambda)=: g\left(\lambda, \lambda-\hbar\left(h^{(1)}+h^{(2)}\right)\right) g^{-1}\left(\lambda-\hbar h^{(2)}, \lambda-\hbar\left(h^{(1)}+h^{(2)}\right)\right) \otimes \\
\otimes g\left(\lambda, \lambda-\hbar\left(h^{(1)}+h^{(2)}\right)\right) g^{-1}\left(\lambda, \lambda-\hbar h^{(2)}\right): .
\end{array}
$$

Let us now calculate the quasi-classical limit of $J(\lambda)$. This means, having $J(\lambda)=1+\hbar \rho(\lambda)+O\left(\hbar^{2}\right)$ to calculate the classical dynamical $r$-matrix $r(\lambda):=$ $\rho^{21}(\lambda)-\rho(\lambda)$. This $r$-matrix is given by the following proposition.

Proposition 3.6. Let $\left\{y_{i}\right\}$ be a basis of $\mathfrak{a}$ and $\left\{y^{i}\right\}$ the dual basis of $\mathfrak{a}^{*}$. Let $g(\lambda, \lambda)=\gamma(\lambda)$. Then

$$
r(\lambda)=\sum_{i} \frac{\partial \gamma(\lambda)}{\partial y^{i}} \gamma^{-1}(\lambda) \wedge y_{i}
$$

Proof. The proof is by a direct calculation. 
Example 3.7. Consider $G=G L(n) \ltimes \mathbb{C}^{n}, \mathfrak{g}=\operatorname{Lie}(G)$, and $\mathfrak{a}=\mathbb{C}^{n}$. Then for any function $f$ whose Jacobian does not vanish identically, we can define the adjoint $g(\lambda, \mu)^{*}: \mathfrak{a}^{*} \rightarrow \mathfrak{a}^{*}$ of $g(\lambda, \mu)$ (which is regarded as an element of $G L(n) \subset G)$ by

$$
g(\lambda, \mu)^{*}=\sum_{m \geq 1} f^{(m)}(\lambda)(\mu-\lambda)^{m-1} / m !
$$

which satisfies the required conditions (here $f^{(m)}(\lambda):\left(\mathfrak{a}^{*}\right)^{\otimes m} \rightarrow \mathfrak{a}^{*}$ is a symmetric linear map). In particular, $g(\lambda, \lambda)=f^{\prime}(\lambda)^{*}: \mathfrak{a}^{*} \rightarrow \mathfrak{a}^{*}$. Thus, we have:

$$
r(\lambda)=r_{n, f}(\lambda)=\sum_{i} \frac{\partial f^{\prime}(\lambda)^{*}}{\partial y^{i}}\left(f^{\prime}(\lambda)^{*}\right)^{-1} \wedge y_{i} .
$$

In particular, for $n=1$ we have a basis $\{X, Y\}$ of $\mathfrak{g}$, where $X$ generates $g l(1)$ and $Y$ generates $\mathfrak{a}=\mathbb{C}$, with $[X Y]=Y$. Then

$$
r_{1, f}(\lambda)=\frac{f^{\prime \prime}(\lambda)}{f^{\prime}(\lambda)} X \wedge Y
$$

This classical $r$-matrix was considered by $\mathrm{Xu}$ in $[\mathrm{X}]$.

In this case, we have $g(\lambda, \mu)=\left(\frac{f(\lambda)-f(\mu)}{\lambda-\mu}\right)^{X}$, and the twist has the form

$$
\begin{gathered}
=:\left(\frac{f(\lambda)-f\left(\lambda-\hbar\left(Y^{(1)}+Y^{(2)}\right)\right)}{Y^{(1)}+Y^{(2)}} \frac{Y^{(1)}}{f\left(\lambda-\hbar Y^{(2)}\right)-f\left(\lambda-\hbar\left(Y^{(1)}+Y^{(2)}\right)\right)}\right)^{X^{(1)}} \\
\quad \times\left(\frac{f(\lambda)-f\left(\lambda-\hbar\left(Y^{(1)}+Y^{(2)}\right)\right)}{Y^{(1)}+Y^{(2)}} \frac{Y^{(2)}}{f(\lambda)-f\left(\lambda-\hbar Y^{(2)}\right)}\right)^{X^{(2)}}
\end{gathered}
$$

where for any monomial $F$ in $X^{i}$ and $Y^{i}$, the expression : $F$ : means the monomial $F$ in which all the $Y$-factors have been moved to the right from the $X$ factors.

Remark 3.8. We note that we have given here a quantization for the dynamical $r$-matrices $r_{n, f}(\lambda)$. As far as we know, such a quantization was previously unknown, even for $n=1$. To be more specific, the paper $[\mathrm{X}]$ constructs a quantization of a vast collection of skew-symmetric dynamical $r$-matrices, but does not cover the case of $r_{1, f}$, except for some specific $f$. In fact, the above constructions were motivated by P. Xu's question "how to quantize $r_{1, f}$ ?"

Remark 3.9. As a special case of $r_{1, f}$, one can consider $f(\lambda)=e^{\lambda}$. In this case, $r_{1, f}$ is a constant $r$-matrix $X \wedge Y$. This $r$-matrix can be quantized by the well known Jordanian twist [GGS], but here we have quantized it as a dynamical $r$-matrix, i.e., preserving the weight zero condition. It is easy to see that the quantization we constructed is also constant (i.e., is a usual twist), but it has zero weight with respect to $Y$ and therefore is more complicated than the usual Jordanian twist. The existence of such "zero weight" quantization follows from $[\mathrm{EK}]$, but here we wrote it explicitly. 


\section{Completely degenerate dynamical $r$-matrices}

Let $\mathfrak{g}$ be a finite-dimensional Lie algebra, and $\mathfrak{a} \subset \mathfrak{g}$ be an Abelian Lie subalgebra. In the following, by functions on $\mathfrak{a}^{*}$ we will mean holomorphic functions defined near $0 \in \mathfrak{a}^{*}$.

Let $\left\{y_{i}\right\}_{i=1}^{n}$ be a basis of $\mathfrak{a}$ and $\left\{y^{i}\right\}_{i=1}^{n}$ be the dual basis of $\mathfrak{a}^{*}$. Let $r$ : $\mathfrak{a}^{*} \rightarrow \wedge^{2} \mathfrak{g}$ be a skew-symmetric classical dynamical $r$-matrix, i.e., a zero weight solution of the classical dynamical Yang-Baxter equation

$$
\begin{aligned}
\sum_{i} & \left(y_{i}^{(1)} \frac{\partial r^{23}(\lambda)}{\partial y^{i}}-y_{i}^{(2)} \frac{\partial r^{13}(\lambda)}{\partial y^{i}}+y_{i}^{(3)} \frac{\partial r^{12}(\lambda)}{\partial y^{i}}\right)+ \\
& {\left[r^{12}(\lambda), r^{13}(\lambda)\right]+\left[r^{12}(\lambda), r^{23}(\lambda)\right]+\left[r^{13}(\lambda), r^{23}(\lambda)\right]=0 . }
\end{aligned}
$$

For basic facts about such $r$-matrices and their quantization, see survey [ES2].

Recall $[\mathrm{X}]$ that $r$ is said to be non-degenerate if the projection $\tilde{r}(\lambda)$ of the element $r(\lambda)$ to $\wedge^{2}(\mathfrak{g} / \mathfrak{a})$ is non-degenerate for some $\lambda$. It is shown in $[\mathrm{X}]$ that such an $r$-matrix can be quantized.

This motivates the following definition.

Definition 4.1. We will say that $r$ is completely degenerate if the induced map $\tilde{r}: \mathfrak{a}^{*} \rightarrow \wedge^{2}(\mathfrak{g} / \mathfrak{a})$ is zero.

In this Section, we classify all completely degenerate dynamical $r$-matrices, and use ideas of Section 3 to show that any such $r$-matrix can be quantized. This generalizes Example 3.7.

Remark 4.2. We note that if $\mathfrak{a}$ has an invariant complement in $\mathfrak{g}$ then it follows from [ES3] that any completely degenerate dynamical $r$-matrix $r: \mathfrak{a}^{*} \rightarrow \wedge^{2} \mathfrak{g}$ is gauge equivalent to zero. However, if such a complement does not exist then the theory of [ES3] does not apply, and in particular there are many nontrivial examples of completely degenerate $r$-matrices (for instance, $r_{n, f}$ considered in the previous section).

Before stating the result, we need to introduce some notation. Let us denote by $\mathfrak{n}(\mathfrak{a})=\{x \in \mathfrak{g} \mid[x, \mathfrak{a}] \subset \mathfrak{a}\}$ the normalizer of $\mathfrak{a}$ in $\mathfrak{g}$, and let $N(\mathfrak{a})$ be the simply connected Lie group corresponding to $\mathfrak{n}(\mathfrak{a})$. We have a natural homomorphism $N(\mathfrak{a}) \rightarrow G L(\mathfrak{a})$, which we will denote by $\gamma \rightarrow \bar{\gamma}$.

Theorem 4.3. $\quad$ (i) Let $\gamma: \mathfrak{a}^{*} \rightarrow N(\mathfrak{a})$ be a function, such that $\bar{\gamma}(\lambda)^{*}=f^{\prime}(\lambda)$ for some function $f: \mathfrak{a}^{*} \rightarrow \mathfrak{a}^{*}$. Then the function

$$
r_{\gamma}(\lambda)=\sum_{i} \frac{\partial \gamma(\lambda)}{\partial y^{i}} \gamma(\lambda)^{-1} \wedge y_{i},
$$

is a completely degenerate dynamical $r$-matrix.

(ii) Every completely degenerate skew-symmetric classical dynamical r-matrix is equal to (23) for some function $\gamma: \mathfrak{a}^{*} \rightarrow N(\mathfrak{a})$. Moreover, $r_{\gamma_{1}}$ is gauge equivalent to $r_{\gamma_{2}}$ in the sense of [ES3, p.3] if and only if $\bar{\gamma}_{1}=\bar{\gamma}_{2}$. 
(iii) Every completely degenerate skew-symmetric classical dynamical $r$-matrix can be quantized.

The rest of this Section is the proof of the theorem.

Proof. Let us first prove (i). Let $r$ be given by (23). First of all, it is easy to check that $r$ has zero weight. Namely, the zero weight condition coincides with the cross-derivative condition for $\bar{\gamma}(\lambda)^{*}$, which is equivalent to the condition that $\bar{\gamma}(\lambda)^{*}=f^{\prime}(\lambda)$ for some $f$. It is also clear that $\tilde{r}=0$. So it remains to check the classical dynamical Yang-Baxter equation for $r$.

Let us write

$$
r(\lambda)=\sum_{i} p_{i}(\lambda) \wedge y_{i}
$$

with $p_{i}(\lambda)=\frac{\partial \gamma(\lambda)}{\partial y^{i}} \gamma(\lambda)^{-1} \in \mathfrak{g}$. Then it is straightforward to check that the zero weight condition for $r$ translates into

$$
\sum_{i} y_{i} \otimes\left[y, p_{i}(\lambda)\right]=\sum_{i}\left[y, p_{i}(\lambda)\right] \otimes y_{i}
$$

for all $y \in \mathfrak{a}$. Using this identity we can write the classical dynamical YangBaxter equation for $r$ in terms of functions $p_{i}(\lambda)$ as

$$
\begin{aligned}
\sum_{i j}\left(\frac{\partial p_{j}(\lambda)}{\partial y^{i}}-\right. & \left.\frac{\partial p_{i}(\lambda)}{\partial y^{j}}+\left[p_{i}(\lambda), p_{j}(\lambda)\right]\right) \otimes y_{i} \otimes y_{j} \\
-y_{i} \otimes & \left(\frac{\partial p_{j}(\lambda)}{\partial y^{i}}-\frac{\partial p_{i}(\lambda)}{\partial y^{j}}+\left[p_{i}(\lambda), p_{j}(\lambda)\right]\right) \otimes y_{j} \\
& +y_{i} \otimes y_{j} \otimes\left(\frac{\partial p_{j}(\lambda)}{\partial y^{i}}-\frac{\partial p_{i}(\lambda)}{\partial y^{j}}+\left[p_{i}(\lambda), p_{j}(\lambda)\right]\right)=0
\end{aligned}
$$

This equation is clearly satisfied, since $p_{i}=\frac{\partial \gamma(\lambda)}{\partial y^{i}} \gamma(\lambda)^{-1}$. So statement (i) is proved.

Let us now prove (ii). Let $r$ be any completely degenerate dynamical $r$-matrix.

Lemma 4.4. We have $r(\lambda) \in \mathfrak{n}(\mathfrak{a}) \wedge \mathfrak{a}$.

Proof. Clearly, $r(\lambda) \in \mathfrak{g} \wedge \mathfrak{a}$. Write $r$ in the form (24). Since $r$ has zero weight, the projection

$$
\hat{r}(\lambda)=\sum_{i} \hat{p}_{i}(\lambda) \otimes y_{i}, \quad \text { where } \quad \hat{p}_{i}(\lambda) \in \mathfrak{g} / \mathfrak{a},
$$

of $r(\lambda)$ on $(\mathfrak{g} / \mathfrak{a}) \otimes \mathfrak{a}$ also has zero weight. This implies that $\left[y_{j}, \hat{p}_{i}(\lambda)\right]=0$ in $\mathfrak{g} / \mathfrak{a}$ for all $i, j$, therefore $\hat{p}_{i}(\lambda) \in \mathfrak{n}(\mathfrak{a}) / \mathfrak{a}$, and hence $p_{i}(\lambda) \in \mathfrak{n}(\mathfrak{a})$.

Now, as we explained above, the classical dynamical Yang-Baxter equation for $r$ and the zero weight condition reduce to equations (26) and (25) respectively. 
In particular, we have

$$
\frac{\partial \hat{p}_{j}(\lambda)}{\partial y^{i}}-\frac{\partial \hat{p}_{i}(\lambda)}{\partial y^{j}}+\left[\hat{p}_{i}(\lambda), \hat{p}_{j}(\lambda)\right]=0 .
$$

This implies that the differential equations

$$
\frac{\partial \hat{\gamma}(\lambda)}{\partial y^{i}}=\hat{p}_{i}(\lambda) \hat{\gamma}(\lambda)
$$

are compatible and have a unique solution $\hat{\gamma}: \mathfrak{a}^{*} \rightarrow N(\mathfrak{a}) / \exp (\mathfrak{a})$ with the initial condition $\hat{\gamma}(0)=1$.

Let $\gamma: \mathfrak{a}^{*} \rightarrow N(\mathfrak{a})$ be an arbitrary lift of $\hat{\gamma}$. Then

$$
r(\lambda)=\sum_{i} \frac{\partial \gamma(\lambda)}{\partial y^{i}} \gamma(\lambda)^{-1} \wedge y_{i}+\sum_{i j} C_{i j}(\lambda) y_{i} \wedge y_{j}
$$

where the second sum is a 2-form. It follows from the classical dynamical YangBaxter equation that $C$ is a closed form. Therefore, $r$ is gauge equivalent to (23), as desired. This proves the first statement of (ii).

To prove the second statement of (ii), it is sufficient to recall that gauge transformations are functions from $\mathfrak{a}^{*}$ to the centralizer $Z(\mathfrak{a})$ of $\mathfrak{a}$ in $N(\mathfrak{a})$. Now, the action of such a function $g$ on $r_{\gamma}$ is given by $\gamma \rightarrow g \gamma$. This implies easily the second statement of (ii), since the image of $N(\mathfrak{a})$ in $G L(\mathfrak{a})$ is exactly $N(\mathfrak{a}) / Z(\mathfrak{a})$.

Finally, let us prove (iii). First of all, as we already mentioned in the proof of (i), the zero weight property of $r$ implies that $\bar{\gamma}(\lambda)^{*}=f^{\prime}(\lambda)$ for some function $f: \mathfrak{a}^{*} \rightarrow \mathfrak{a}^{*}$. Now, for $\lambda \in \mathfrak{a}^{*}$ define the element

$$
x(\lambda)=\sum_{m \geq 1} \frac{(-1)^{m-1}}{m !} \gamma^{(m-1)}(\lambda)\left(\hbar h^{(1)}\right)^{m-1}
$$

of the group $\tilde{H}^{\times}$for $H=U(\mathfrak{n}(\mathfrak{a}))[[\hbar]]$. Then it is easy to check that $x(\lambda) h^{(1)}=$ $\hbar^{-1}\left(f(\lambda)-f\left(\lambda-\hbar h^{(1)}\right)\right) x(\lambda)$. Therefore, by Section $3, x(\lambda)$ is a vertex-IRF transformation of $1 \otimes 1$. Hence $J(\lambda)=\Delta(x(\lambda)) x^{2}(\lambda)^{-1} x^{1}\left(\lambda-h^{(2)}\right)^{-1}$ is a dynamical twist. It is easy to check directly that the quasi-classical limit of $J(\lambda)$ is $r(\lambda)$. The theorem is proved.

Remark 4.5. We warn the reader that $x(\lambda)$ is not quasi-grouplike, and hence for the examples of Section 3, the quantization constructed here coincides with the quantization by "quasi-grouplike elements" of Section 3 only up to gauge transformations.

\section{Acknowledgments}

We are grateful to Eric Buffenoir, Phillippe Roche, and Ping Xu for useful discussions. In particular, we are grateful to Ping $\mathrm{Xu}$ for asking the question that led us to the results of Sections 3 and 4. The second author thanks MIT for the warm hospitality during his visit. The authors were partially supported by the NSF grant DMS-9988796. 


\section{References}

[BBB] O. Babelon, D. Bernard, and E. Billey, A Quasi-Hopf algebra interpretation of quantum 3-j and 6-j symbols and difference equations, Phys. Lett. B 375 (1996), 89-97.

[BNSz] G. Böhm, F. Nill, and K. Szlachányi, Weak Hopf algebras I. Integral theory and $C^{*}$ structure, J. Algebra, 221 (1999), 385-438.

[EK] P. Etingof and D. Kazhdan, Quantization of Lie bialgebras. II, Selecta Math. (N.S.) 4 (1998), 213-231.

[EN1] P. Etingof, D. Nikshych, Dynamical quantum groups at roots of 1, Duke Math. J., 108 (2001), to appear, math.QA/0003221.

[EN2] P. Etingof, D. Nikshych, Dynamical twists in finite groups, Internat. Math. Res. Notices, to appear, math.QA/0101046.

[ES1] P. Etingof, O. Schiffmann, A link between two elliptic quantum groups, Asian J. Math. 2 (1998), 345-354.

[ES2] L Lectures on the dynamical Yang-Baxter equations, preprint, math.QA/9908064 (1999).

[ES3] - On the moduli space of classical dynamical r-matrices, preprint, math.QA/0005282 (2000).

[EV] P. Etingof, A. Varchenko, Exchange dynamical quantum groups, Comm. Math. Phys. 205 (1999), 19-52.

[GGS] M. Gerstenhaber, A. Giaquinto, and S. Schack, Quantum symmetry, in : "Quantum groups" (Leningrad, 1990), 9-46, Lecture Notes in Math. 1510, Springer, Berlin, 1992.

[F] G. Felder, Elliptic quantum groups, XIth International Congress of Mathematical Physics (1994), 211-218, Internat. Press, Cambridge, 1995.

[Has] K. Hasegawa, L-operator for Belavin's R-matrix acting on the space of theta functions, J. Math. Phys. 35 (1994), 6158-6171.

[Ho] T. Hodges, Generating functions for coefficients of Cremmer-Gervais R-matrices, math.QA/9903113.

[JOKS] M. Jimbo, S. Odake, H. Konno, and J. Shiraishi, Quasi-Hopf twistors for elliptic quantum groups, Transform. Groups 4 (1999), 303-327.

[KZ] I.M. Krichever and A. Zabrodin, Spin generalization of the Ruijsenaars-Schneider model, the nonabelian two-dimensionalized Toda lattice, and representations of the Sklyanin algebra, (Russian) Uspekhi Mat. Nauk 50 (1995), 3-56, translation in Russian Math. Surveys 50 (1995), 1101-1150.

[M] S. Majid, Foundations of quantum group theory, Cambridge University Press, 1995.

[X] P. Xu, Triangular dynamical r-matrices and quantization, preprint, math.QA/0005006.

Department of Mathematics, Massachusetts Institute of Technology, 77 Massachusetts Avenue, Cambridge, MA 02139.

Department of Mathematics, Columbia University, 2990 Broadway, New York, Ny 10027-4307.

E-mail address: etingof@math.mit.edu

Dept. of Mathematics, UClA, 405 Hilgard Avenue, Los Angeles, CA 90095-1555.

Dept. of Mathematics, Mit, 77 Massachusetts Avenue, Room 2-130, Cambridge, MA 02139-4307.

E-mail address: nikshych@math.ucla.edu 